Preface

\title{
Anticoagulant Therapy: Present and Future
}

\author{
Emmanuel J. Favaloro, PhD, FFSc (RCPA) ${ }^{1}$ \\ ${ }^{1}$ Department of Haematology, Institute of Clinical Pathology and \\ Medical Research (ICPMR), Westmead, Australia \\ Semin Thromb Hemost 2015;41:109-112.
}

Welcome to a very special issue of Seminars in Thrombosis $\mathcal{E}$ Hemostasis. This issue, on the topic of "Anticoagulant therapy: present and future," is guest edited by Job Harenberg, a longtime member of the editorial board and supporter of the journal. Indeed, Dr. Harenberg most recently wrote a historical sketch for the journal around the many meetings that he organized on the anticoagulant and nonanticoagulant actions of glycosaminoglycans, a family of compounds that includes heparin. ${ }^{1}$ The journal's founding Editor-in-Chief, Eberhard Mammen, was in attendance at several of these meetings. This period of time represents a special phase for Dr. Harenberg, who is approaching a stage of forced semiretirement, given his upcoming 65th birthday and an unfortunate compulsory retirement from university duties. Dr. Harenberg continues, nonetheless, to be an active contributor to the field of thrombosis and hemostasis, as attested by the current bumper issue that he has assembled. Dr. Harenberg also clearly expresses his deep appreciation to the Dietmar Hopp foundation, which has provided substantial support for his research activities over many years as well as facilitating procurement of additional copies of the current issue for advancement of knowledge in the field.

The initial contribution to this issue is by Agnelli et al, ${ }^{2}$ on the methodological quality of studies assessing thromboprophylaxis with low-molecular-weight heparin (LMWH), which represent the standard of care for prophylaxis of venous thromboembolism (VTE). The authors have reviewed the evidence supporting the use of the different LMWHs in VTE prophylaxis, in different clinical settings, and analyzed this progression over time. They conclude that the quality of studies supporting LMWH for VTE prophylaxis in different clinical settings is not homogeneous and inferior for studies performed before the year 1990. Also, clinical interchangeability of LMWHs in clinical practice remains a critical issue, and they propose that the selection of a product should be based on evidence available for each agent, and for each clinical indication derived from the clinical trials.

Address for correspondence Emmanuel J. Favaloro, PhD, FFSc (RCPA), Department of Haematology, Institute of Clinical Pathology and Medical Research (ICPMR), Westmead Hospital, Westmead, Australia (e-mail: emmanuel. favaloro@health.nsw.gov.au).
The next article is provided by Prandoni et $\mathrm{al}^{3}$ on the impact of residual vein thrombosis (RVT) on the long-term outcome of patients with deep venous thrombosis (DVT), treated with conventional anticoagulation, which is currently largely unknown. These authors have therefore assessed the incidence of recurrent VTE, postthrombotic syndrome (PTS), arterial thrombotic events, and cancer in patients with DVT, with and without RVT, and using a dataset of more than 800 consecutive patients with acute proximal DVT who had conventional anticoagulation. RVT was detected in almost half of the patients, and was more likely in males, in patients with previous VTE and in those with extensive thrombosis. During the 3-year follow-up recurrent VTE, PTS, arterial thrombosis, and cancer developed in twice the number of patients with, compared with without, RVT. They conclude that studies are needed to address the impact of the nonvitamin $\mathrm{K}$ antagonist (VKAs) oral anticoagulants (NOACs) on the development of RVT as well as the long-term complications of DVT.

The third contribution, by Dahl et $a l,{ }^{4}$ discusses arterial and venous thrombosis following trauma and major orthopedic surgery, as well as the molecular mechanisms and strategies for intervention. A variety of harmful effects can be triggered by trauma and major orthopedic surgery, with thrombin being one of the key players involved in such adverse processes, and DVT and pulmonary embolism (PE) being potential clinical outcomes. Protecting patients from VTE has been the main goal of preventive strategies, including thromboprophylaxis aiming in part to control thrombin generation and activation. However, recent years have seen expansion of knowledge related to cell destruction and release of substances that may cause organ damage, including small molecules such as RNAs and histones, which may cause additional cell destruction and organ damage at distal sites if released in huge amounts and disseminated systemically. Accordingly, there is an unmet need for therapies that prevent both vascular events and organ deterioration, and this article briefly reviews
Copyright (C) 2015 by Thieme Medical Publishers, Inc., 333 Seventh Avenue, New York, NY 10001, USA. Tel: +1(212) 584-4662.
DOI http://dx.doi.org/ 10.1055/s-0035-1546828. ISSN 0094-6176.
Therapy: Present and Future; Guest Editor: Job Harenberg, MD. 
molecular mechanisms associated with the occurrence of vascular events and cellular destruction in patients with major bone damage caused by trauma.

This issue of Seminars in Thrombosis \& Hemostasis then turns to more intensive discussions on the NOACs, a discussion initiated by Senoo and Lip, ${ }^{5}$ who discuss the comparative efficacy and safety of the NOACs for patients with nonvalvular atrial fibrillation. The NOACs include the thrombin inhibitor (dabigatran) and the direct factor Xa inhibitors (rivaroxaban, apixaban, and edoxaban). These have been shown to be at least as efficacious and safe at fixed daily doses as conventional international normalized ratio adjusted oral anticoagulants, such as the VKAs (including warfarin), for stroke prevention in patients with nonvalvular atrial fibrillation. Each NOAC has various advantages and specific features, and therefore, decisions regarding appropriate stroke prevention require individual assessment of stroke and bleeding risk on anticoagulation with VKA therapy and NOACs when starting on any of these drugs. This review briefly describes the results of clinical randomized trials for these four NOACs, and discusses how they might impact clinical practice and choice of anticoagulants in atrial fibrillation patients. The review also examines the differences of the proposed management of antithrombotic therapy in several international guidelines and pragmatic issues of NOACs for stroke prophylaxis.

Prandoni et $\mathrm{al}^{6}$ then discuss the incidence of arterial embolism in patients treated with conventional or NOACs for VTE. First, they consider that the classically conceived separate nature of venous versus arterial thrombotic disorders can be debated, since patients with VTE also have an increased risk of subsequent symptomatic arterial cardiovascular events. Accordingly, there is potential for any ("old" or "new") antithrombotic drug to impact on the development of both venous and arterial cardiovascular events. However, the results from recent randomized clinical trials suggest that the likelihood of arterial cardiovascular events in patients on NOACs is unlikely to differ from that of patients receiving conventional anticoagulation.

Next, Schulman ${ }^{7}$ reviews the treatment of VTE with NOACs according to patient risks. Recent developments in antithrombotic therapy have now provided us with many choices for the management of VTE, including unfractionated heparin, LMWH, VKA, and inhibitors against factor Xa or against thrombin. The factors that influence decisions on which to use should be efficacy, safety, convenience and when appropriate also patient values and preferences. For most patients there is a strong evidence regarding safety from large randomized trials, but this does not apply to all patients. In this review, the choice of treatment is focused on the risks that are known or can be predicted for the individual patient, and discusses the three principal decision points where tailoring of individual therapy are considered-at diagnosis, for hospitalized patients at the time of discharge, and finally after 3 to 6 months.

Zolfaghari et $\mathrm{al}^{8}$ consider an aspect of such decisions, namely, considerations to continue anticoagulation with the different types of oral anticoagulants based on the patientś preference. In particular, patients with indications for anticoagulation may prefer treatment with either a VKA or NOAC. The authors have therefore developed a questionnaire that may help to identify the preference of patients for one of these agents, as well as to potentially continue or to change the anticoagulant. These authors previously reported in this journal on the development of this tool. ${ }^{9}$

Continuing the NOAC "story," Riva and Ageno ${ }^{10}$ discuss the pros and cons of VKAs and NOACs. The NOACs have emerged as alternative options for the prevention and treatment of VTE and for the prevention of stroke and systemic embolism in patients with nonvalvular atrial fibrillation. The NOACs have several practical advantages over VKAs, such as the rapid onset/offset of action, the lower potential for food and drug interactions and the predictable anticoagulant response. However, the VKAs currently have a broader spectrum of indications, a standardized monitoring test, and established reversal strategies, and there also remain some populations for whom the VKAs remain the most appropriate anticoagulant drug.

McMahon and Kwaan ${ }^{11}$ then cover a similar, but not identical, area to discuss what we have learned since the arrival of the NOACs. In particular, although these agents have several advantages over VKAs, lower variability of pharmacological actions such as fewer drug interactions, and improved safety, especially intracranial hemorrhage, several issues have arisen with their increasing usage, with concerns over monitoring, and reversal, being predominant. This article, therefore, discusses their efficacy, bleeding risk, and the recognition of vulnerable populations in which determination of the anticoagulant effect is needed. Moreover, the current approach to reversing the drug action is also discussed, as is the change in the approach to future drug design.

Chin ${ }^{12}$ then discusses the question of which patients may benefit from dose-adjustment of the NOACs. Dose-adjustment of the NOAC takes into account the presence of particular patient characteristics that may alter NOAC concentrations, including the presence of renal impairment and concomitant drugs affecting proteins involved with the transport and metabolism of the NOAC. Plasma NOAC concentrations reflect the anticoagulant activity; some studies have shown some correlation with the risks of adverse events, while others have not, and the association is debated by workers in the field. However, Dr. Chin advises that dose-adjustment based on characteristics before dosing assumes that the patient has drug oral availability and clearance at the mean of patients with these characteristics. Therefore, direct quantification of NOAC concentrations, for example, through the use of calibrated coagulation assays, is likely to add further benefit for specific patient populations and clinical circumstances by the individualization of dosing.

This brings us to the topic of laboratory and nonlaboratory-aided measurements of the NOACs whenever required, and this is covered by two separate articles in this issue of Seminars in Thrombosis and Hemostasis. First, Favaloro and Lippi ${ }^{13}$ review the challenge of laboratory testing in the era of NOACs, with the aim to provide a practical guide to measuring their activity and avoiding diagnostic errors. The industryguided suggestion that NOACs do not require laboratory 
monitoring is countered by the ongoing recognition that laboratory testing for drug effects is needed in many situations. Moreover, since these agents "do not require" laboratory monitoring, some clinicians inappropriately take this to mean that they do not affect hemostasis tests. This review reports on the laboratory studies that have evaluated the NOACs against a wide range of laboratory assays to assess the utility for qualitative or quantitative measurements of these drugs, as well as interferences that may cause misdiagnosis of hemostatic defects, and extends on findings previously reported by them. ${ }^{14-16}$

Second, Harenberg et al $^{17}$ provide insight into the use of patients' serum or urine as easily accessible samples for the measurement of the NOACs. Plasma samples are not always available, and sometimes nonlaboratory testing may be useful. Such methods should give results rapidly within minutes, should be easy to perform, specific, and sensitive. The authors therefore describe various procedures that may be undertaken, either within the laboratory or outside, using plasma samples, or even alternatives blood samples anticoagulated with ethylenediaminetetraacetic acid or with serum. NOAC can also be determined in urine samples due to their renal elimination. Although quantitative methods are often preferable to qualitative methods, the latter may be advantageous in some situations, in particular as point-of-care tests, where the presence and absence of NOAC can be identified with the naked eye after a few minutes. Finally, new assays such as a semiquantitative determination in urine samples and measurement using other sample matrices are currently under development. Dr. Harenberg and colleagues have reported extensively on this topic in the past. ${ }^{18-22}$

The next two contributions are related to therapeutic, or potential therapeutic, approaches in the case of cancerassociated treatments. First, $\mathrm{Wu}$ and Lee $^{23}$ discuss the treatment of cancer-associated thrombosis in the era of the NOACs. Despite advances in therapy using LMHW, both VTE recurrence and clinically relevant bleeding while on therapeutic anticoagulation occur at high rates in cancer patients. There are many attractive features of the NOACs, including the convenience and simplicity of administration, but there are also several limitations such as dependency on gastrointestinal absorption, renal clearance, and some significant drug-drug interactions. At the moment, the use of these newer oral agents in cancer patients is not recommended as their safety and efficacy are not yet established and the complexity of these patients warrants further cancer-specific clinical trials.

Second, Gerber et $a^{24}$ discuss the potential future of targeting of heparanase to reduce metastatic progression. Heparanase is an endo- $\beta$-glucuronidase that enzymatically cleaves heparan sulfates and similar structures. Heparanase expression levels by tumors are correlated with cell invasion, angiogenic activity, and poor prognosis, also heparanase possess protumorigenic effects independent of its enzymatic activity. Of relevance, the integrin VLA-4 appears to be involved in tumorigenicity via a heparanase/integrin activation axis, and heparin interferes with this activation process. $\mathrm{LMWH}$, for example, appears capable of blockade of hepar- anase, with functional consequences of reduced VLA-4 binding. Thus, latent heparanase appears to be a novel, so far unnoticed target of heparin, underlying its antimetastatic activity, and offering insight into possible future potential therapies to reduce metastatic progression.

This issue of Seminars in Thrombosis \& Hemostasis concludes with a look to possible future "next generation" antithrombotic therapies, namely, antisense therapy, such as that against coagulation factor XI, by Lippi et al. ${ }^{25}$ Antisense oligonucleotides are relatively short single-stranded nucleic acid sequences, which hybridize with a target mRNA and suppress protein synthesis. Coagulation factor XI is a key player in blood coagulation, and thus represents a potential target for antisense therapy. The available evidence reviewed in this article suggests that factor XI antisense oligonucleotides may be more effective than conventional anticoagulants in preventing the onset and propagation of thrombosis, do not require factor measurement since the reduction of mRNA synthesis appears dose-dependently, robustly, and stably decreased for 3 to 5 weeks after the end of administration, with an incidence of major bleeding that is at least not greater than that associated with warfarin or LMWH therapy. Despite conceptual simplicity, rational design, and relatively inexpensive cost, the authors further advise that the preliminary findings in animal models and in patients undergoing knee surgery need to be validated in other prospective trials and cost-effective analyses before this treatment option can be advocated as a new paradigm for the prevention and treatment of VTE.

To conclude, I would like to sincerely thank the Guest Editor for this issue of Seminars in Thrombosis $\mathcal{E}$ Hemostasis, Job Harenberg, for compiling this most interesting issue, as well as all the authors to this issue for their original and comprehensive contributions. I hope that the readership finds the issue to be of substantial interest, and I look forward to the next issue prepared by Dr. Harenberg for this journal. May his "semiretirement" keep him very busy.

\section{References}

1 Harenberg J, Casu B, Krämer R, Torri G, Naggi A, Krämer S. Glycosaminoglycans: anticoagulant and nonanticoagulant actions: a short history of symposia held at villa vigoni. Semin Thromb Hemost 2014;40(8):831-836

2 Agnelli G, Prandoni P, Di Minno G, et al. Thromboprophylaxis with low-molecular-weight heparins: an assessment of the methodological quality of studies. Semin Thromb Hemost 2015;41(2):113-132

3 Prandoni P, Lensing AWA, Prins MH, et al. The impact of residual thrombosis on the long-term outcome of patients with deep venous thrombosis treated with conventional anticoagulation. Semin Thromb Hemost 2015;41(2):133-140

4 Dahl OE, Harenberg J, Wexels F, Preissner KT. Arterial and venous thrombosis following trauma and major orthopedic surgery: molecular mechanisms and strategies for intervention. Semin Thromb Hemost 2015;41(2):141-145

5 Senoo K, Lip GYH. Comparative efficacy and safety of the non-vitamin $\mathrm{K}$ antagonist oral anticoagulants for patients with non-valvular atrial fibrillation. Semin Thromb Hemost 2015;41(2):146-153

6 Prandoni P, Milan M, Barbar S, et al. Incidence of arterial embolism in patients on treatment with old and new anticoagulants for venous thromboembolism. Semin Thromb Hemost 2015;41(2):154-159 
7 Schulman S. Treatment of venous thromboembolism with new oral anticoagulants according to patient risks. Semin Thromb Hemost 2015;41(2):160-165

8 Zolfaghari S, Harenberg J, Froelich L, et al. Development of recommendations to continue anticoagulation with one of the two types of oral anticoagulants based on the identification of patients' preference. Semin Thromb Hemost 2015;41(2): 166-177

9 Zolfaghari S, Harenberg J, Froelich L, Wehling M, Weiss C. Development of a tool to identify patients' preference for vitamin $\mathrm{K}$ antagonist or direct oral anticoagulant therapy. Semin Thromb Hemost 2014;40(1):121-128

10 Riva N, Ageno W. Pros and cons of vitamin K antagonists and nonvitamin K antagonist oral anticoagulants. Semin Thromb Hemost 2015;41(2):178-187

11 McMahon BJ, Kwaan HC. The new or non-vitamin K antagonist oral anticoagulants (NOACs): what have we learned since their debut. Semin Thromb Hemost 2015;41(2):188-194

12 Chin PKL. Which patients may benefit from dose-adjustment of non-vitamin K antagonist oral anticoagulants (NOAC)? Semin Thromb Hemost 2015;41(2):195-207

13 Favaloro EJ, Lippi G. Laboratory testing in the era of the direct or non-Vitamin K antagonist oral anticoagulants (NOACs): a practical guide to measuring their activity and avoiding diagnostic errors. Semin Thromb Hemost 2015;41(2):208-227

14 Lippi G, Favaloro EJ. Recent guidelines and recommendations for laboratory assessment of the direct oral anticoagulants (DOACs): is there consensus? Clin Chem Lab Med 2015;53(2): 185-197

15 Favaloro EJ, Lippi G, Koutts J. Laboratory testing of anticoagulants: the present and the future. Pathology 2011;43(7):682-692
16 Favaloro EJ, Lippi G. The new oral anticoagulants and the future of haemostasis laboratory testing. Biochem Med (Zagreb) 2012; 22(3):329-341

17 Harenberg J, Du S, Krämer S, Weiss C, Krämer R, Wehling M. Patients' serum and urine as easily accessible samples for the measurement of non-vitamin $\mathrm{K}$ antagonist oral anticoagulants. Semin Thromb Hemost 2015;41(2):228-236

18 Harenberg J, Krämer S, Du S, Weiss C, Krämer R. Concept of a point of care test to detect new oral anticoagulants in urine samples. Thromb J 2013;11(1):15

19 Harenberg J, Du S, Krämer S, et al. Novel methods for assessing oral direct factor Xa and thrombin inhibitors: use of point-of-care testing and urine samples. Semin Thromb Hemost 2013;39(1):66-71

20 Harenberg J, Kraemer R. Measurement of the new anticoagulants. Thromb Res 2012;129(Suppl 1):S106-S113

21 Harenberg J, Krämer S, Du S, et al. Measurement of rivaroxaban and apixaban in serum samples of patients. Eur J Clin Invest 2014; 44(8):743-752

22 Harenberg J, Kraemer S, Du S, et al. Determination of direct oral anticoagulants from human serum samples. Semin Thromb Hemost 2014;40(1):129-134

$23 \mathrm{Wu} \mathrm{C}$, Lee AYY. Novel or non-vitamin $\mathrm{K}$ antagonist oral anticoagulants (NOACs) and the treatment of cancer-associated thrombosis. Semin Thromb Hemost 2015;41(2):237-243

24 Gerber U, Hoß SG, Shteingauz A, et al. Latent heparanase facilitates VLA-4 mediated melanoma cell binding and emerges as a relevant target of heparin in the interference with metastatic progression. Semin Thromb Hemost 2015;41(2):244-254

25 Lippi G, Harenberg J, Mattiuzzi C, Favaloro EJ. Next generation antithrombotic therapy: focus on antisense therapy against coagulation factor XI. Semin Thromb Hemost 2015;41(2):255-262 